\title{
ROLE OF MULTI-DETECTOR CT IN THE LOCAL STAGING OF UTERINE MALIGNANCIES
}

\author{
Sameh Mohamed Abdel Wahabl, Lobna Abdel Monem Habib \\ Dayemland Heba Hamdy El Kafrawy ${ }^{2}$
}

\author{
${ }^{\imath}$ Radiodignosis, Department, \\ Faculty of Medicine, Ain Shams \\ University, Radiodignosis Dept \\ Manshyet Elbakry \\ Hospital,Ministry of Health, \\ Cairo, Egypt.
}

Corresponding Author:

Heba Hamdy El Kafrawy

E.mail:

S elkafrawy@yahoo.com

Mobil:01223295862

Received: $12 / 3 / 2020$

Accepted: 8/4/2020

Online ISSN: 2735-3540

\begin{abstract}
:
Background: Multidetector Computed Tomography is a noninvasive and sensitive method for preoperative assessment of uterine neoplasms. Provided CT examination is performed by a specific protocol and image interpretation performed by a specialized radiologist, CT shows good accuracy in the local staging of endometrial and cervical carcinoma.
\end{abstract}

Aim of Work: To assess the role of MDCT in the local staging of uterine malignancies.

Patients and Methods: The study included 51 patients compromised 41 primary and 10 recurrent uterine malignancies that were classified as primary uterine corpus malignancy, primary cervical malignancy and recurrent cases.

Results: The study included 24 female patients known of uterine malignancies that needed staging of their primary disease (14 cases) or required imaging for possible recurrent disease (10 cases). The staging was performed using post contrast CT examination of the abdomen and pelvis and the results were compared with pathological results being the accepted standard for comparison. For cases with advanced malignancy examination under anaesthesia (EUA) was the alternative.

Conclusion: In our study the main concern was to achieve maximum utility of multidetector CT in the local staging of uterine malignancies especially it is a routine tool for screening metastases

Key Words: CT, Malignancies, CIN, ESS, SEER

\section{INTRODUCTION:}

Malignant neoplasms of the female pelvic organs account for almost $15 \%$ of all cancers in women. The most common of these malignancies is uterine cancer, specifically, endometrial cancer ${ }^{(1)}$.

Endometrial cancer is the fourth most frequent cancer in women and now the most common gynaecological cancer in many developed countries; accounting for $6 \%$ of female cancers, following breast, lung, and colorectal cancer. However, it has a favourable prognosis because the majority of patients present at an early stage, resulting in only $3 \%$ of cancer deaths in women ${ }^{(2)}$.Cervical carcinoma accounts for most cases of malignant cervical tumours representing the third most common gynaecologic malignancy ${ }^{(3)}$. The incidence of cervical cancer in Egypt is $0.04 \%$ of the Egyptian woman ${ }^{(4)}$. Nearly $80 \%$ of cervical cancer occurs in the developing countries and most patients are diagnosed with the disease at an advanced stage so staging is usually clinical $^{(5)}$. However, there are significant inaccuracies in the clinical staging system compared to surgical staging, with a considerable error rate $^{(6)}$. In June 2009, the International Federation of 
Gynaecology and Obstetrics (FIGO) introduced the revised staging of cervical carcinoma and although the revised FIGo staging system does not include imaging in the staging of cervical cancer, for the first time the committee encourages the use of imaging techniques ${ }^{(7)}$. In a study to evaluate multislice computed tomography(MSCT) in local staging of endometrial cancer accuracy was proven with high sensitivity and specificity indices ${ }^{(8)}$. Because CT is often the most readily available cross-sectional imaging modality, many female patients undergo $\mathrm{CT}$ as the primary imaging modality, even when US or MR imaging may be more suitable. Therefore, it is important that radiologists get to know the appearance of female pelvic conditions in $\mathrm{CT}^{(9)}$. Although ultra-sonography and magnetic resonance imaging remain the primary imaging modalities for the assessment of most female pelvic disorders, more accurate diagnosis of these disorders at multi-detector CT may obviate additional imaging tests and allow more appropriate management ${ }^{(10)}$.

\section{AIM OF THE WORK:}

To assess the role of MDCT in the local staging of uterine malignancies

\section{PATIENTS AND METHODS:}

Our study was conducted in the National Cancer Institute, Radiology Department. Patients were referred to the radiology unit from the surgery or radiotherapy department for staging of their primary or recurrent disease between $6 / 2015$ and $6 / 2017$.

The study included 51 patients compromised 41 primary and 10 recurrent uterine malignancies that were classified as primary uterine corpus malignancy, primary cervical malignancy and recurrent cases.
The patients' age ranged from 31 to 81 years. The patients' complaint was vaginal bleeding in $>90 \%$ of cases, only a minority had different complaint as suprapubic pain or discharge.

- Patients history included:

1. Age

2. Complaint and its duration

3. Menstual history

4. Parity

Routine laboratory investigation for all patients including complete blood picture, random blood sugar, liver and kidney functions were performed. The normal serum creatinine ranges between 0.6$1.2 \mathrm{mg} / \mathrm{dl}$. For serum Creatinine levels above $2 \mathrm{mg} / \mathrm{dl}$, CT scan with contrast was contraindicated.

\section{Inclusion criteria:}

No age predilection.

- Patients presenting with vaginal bleeding or palpable uterine masses that proved malignant by curettage or smear.

- No previous treatment received (except in recurrent cases)

\section{Exclusion criteria:}

- Contraindication to CT examination: as pregnancy or hypersensitivity to contrast material.

\section{Technique:}

Prior scanning, $1000 \mathrm{ml}$ of oral contrast medium was administrated 3 hours earlier for properbowel opacification. Image acquisition was performed 70 seconds after contrast administration. The contrast agent used was "Telebrex", the dose is $1.1 \mathrm{ml} / \mathrm{kg}$ body weight, with a total dose ranging between 60 to $120 \mathrm{ml}$.

In cases with known sensitivity to contrast medium "Omnipaque" was the alternative agent, the dose is $50-200 \mathrm{~mL}$ (Omni 300); 60-100 mL (Omni 350). The contrast was injected either by the injector or manually. 
The patient was instructed to hold his breath during image acquisition. The scan time lasts about 20 seconds. In case of triphasic study the scan is repeated 4 times, one before injection and the other 3 after injection at the correct timing for each.

All the patients were examined in the supine position. Axial images with a slice thickness of $0.6 \mathrm{~mm}$ were obtained for each case. Coronal and sagittal MPR (multiplanner reconstruction) images were generated from the native axial images on the workstation with a slice thickness of 0.5 $\mathrm{cm}$.

In a sample of 18 patients an experimental analysis was performed where the performed CT examination was extended to include a triphasic study of the pelvis that wasscanned at 20, 70 seconds followed by another delayed scanning at 7 minutes. Such performance had lengthened the examination time and so the precontrast, arterial, venous and delayed images were examined to determine the added benefit of these phases.

As the sample proved no added benefit of precontrast and arterial phases apart from delayed phase which showed excellent ureteric visualization, another 4 patients had an extended study of post contrast CT scan in the venous phase followed by delayed images at 7 minutes to enhance ureteric visualization instead of complete triphasic study.

In two patients sterilised ultrasound gel was injected within vagina; to achieve proper delineation of the disease extent.

Rectal enema was performed for 10 cooperative patients to find out if it has an added value in determining rectal invasion.

\section{RESULTS:}

The study included 51 female patients known of uterine malignancies that needed staging of their primary disease (41 cases) or required imaging for possible recurrent disease (10 cases). The staging was performed using post contrast CT examination of the abdomen and pelvis and the results were compared with pathological results being the accepted standard for comparison. For cases with advanced malignancy examination under anaesthesia (EUA) was the alternative.

\section{Endometrial Cancer Cases:}

Endometrial carcinoma was elicited in 14 patients with age ranging from 36 to 81 . Mean age was 61 and median age was 59 .

The general complaint was vaginal bleeding except for one patient who complained of vaginal discharge.

Nine cases were type I, endometrioid carcinoma, while only one case was clear cell type.

Regarding menstrual history, 13 patients were postmenopausal while only one patient was premenopausal (36 years),

The diameter of the endometrial cancer ranged from 2.9 to $9.2 \mathrm{~cm}$ with a mean of $5.8 \mathrm{~cm}$ while in the pathology the size ranged from 2.5 to $8 \mathrm{~cm}$ with a mean of $5.3 \mathrm{~cm}$.

Six cases had hypo-attenuating masses (appear darker in CT than normal uterus) in relation to normal uterus.

One case had a heterogenous mass, one was a hyper-attenuating mass and the other was iso-attenuating.

\section{Cervical Cancer Cases:}

The study included 27 reported patients of cancer cervix with their ages ranging from 31 to 88 with mean= 52 and median= 51.

The general complaint was vaginal bleeding except for two cases, one had vaginal bleeding with associated left supraclavicular mass and the other had left inguinal mass as the only symptom.

The mass diameter in CT ranged from 3.6 to $10 \mathrm{~cm}$ with a mean of $5.8 \mathrm{~cm}$ and a 
median of $5.3 \mathrm{~cm}$ while in pathology it ranged from 3 to $11 \mathrm{~cm}$ with a mean of $6 \mathrm{~cm}$ and a median of $5 \mathrm{~cm}$.

Fourteen cases had hypoattenuating masses in relation to normal uterus, seven cases had hyper densehyper attenuating and three cases had isodense were isoattenuating masses. Three cases had no masses that could be identified by CT and were considered stage IA or IB according to whether the cancer was recognized clinically or not.

\section{Recurrent Cases:}

\section{Postcontrast CT examination:}

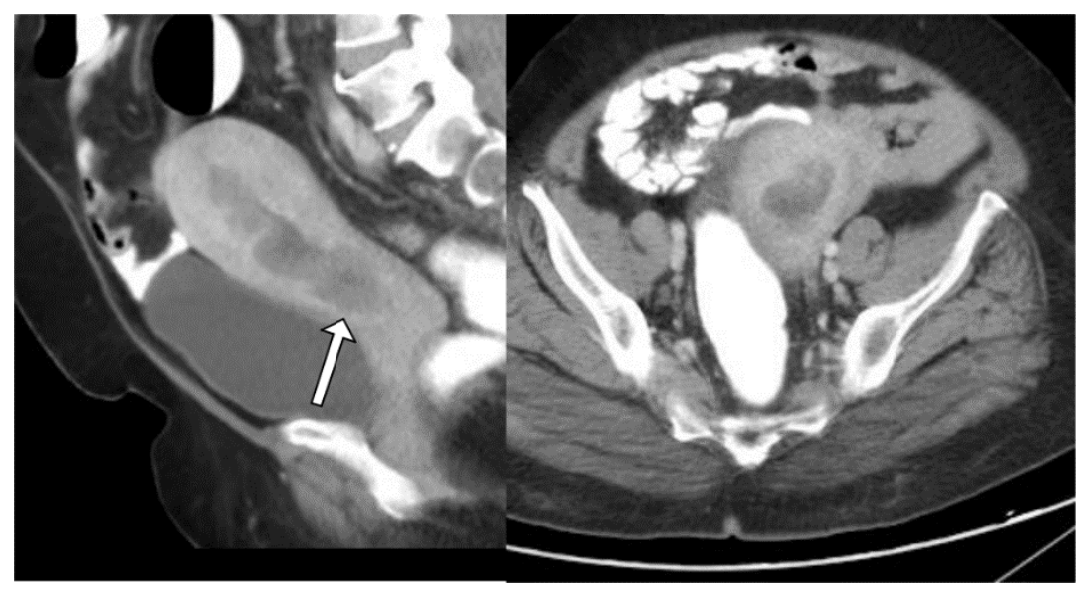

A

B

Figure (1):(A)sagittal,(B)axialimage,showingahypo attenuating endometrial mass invading the myometrium at different levels. Extension of the mass to the cervix is observed in the sagittal view (arrow).

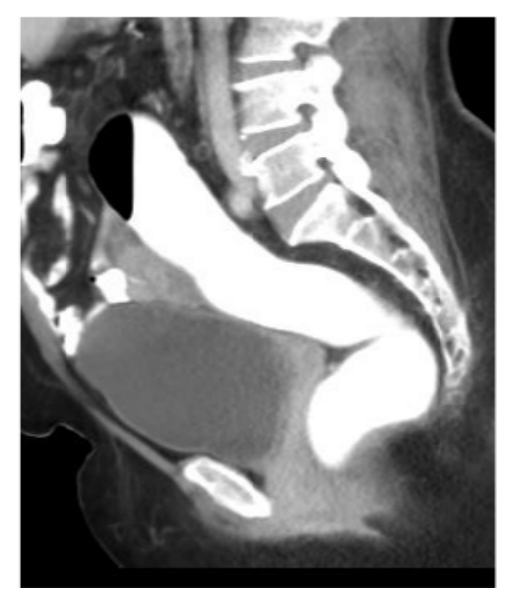

Figure (2): Sagittal view demonstrating free bladder and rectum in relation to mass. rectal enema together with full bladder aided in better visualisation and interpretation. 


\section{Stage: Endometrioid Adenocarcinoma.}

CT stage: II

Pathology stage: II

Case no. 2:41 year old female.

History of premenopausal bleeding of 6 month duration.

\section{Postcontrast CT examination:}

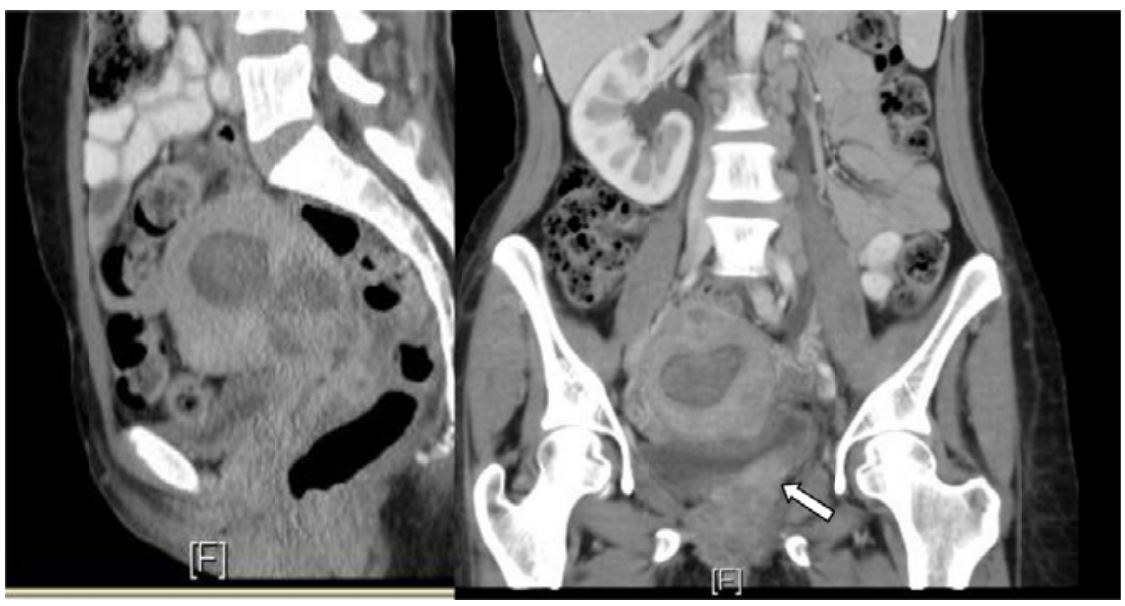

A

B

Figure (3a\&b): Sagittal and coronal images respectively. A heterogeneous cervical mass is seen extending to fundus (A) together with infiltration of the bladder from its inferior aspect causing enhancement of bladder wall (arrow)(B).

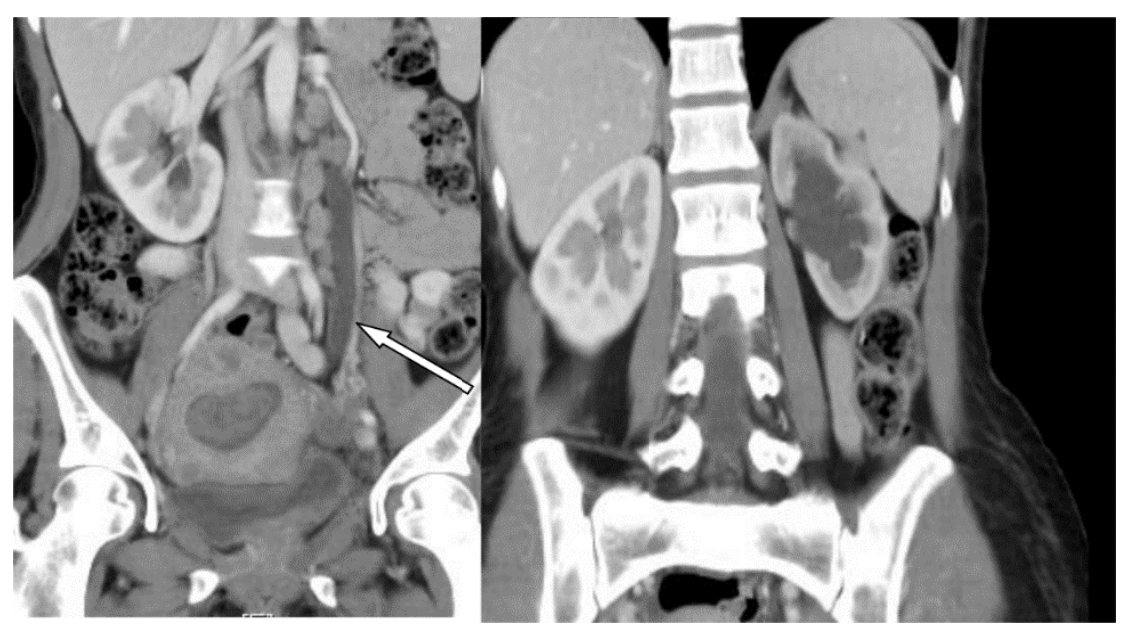

Figure (3a\&b): Coronal images demonstrating, A: left ureteric taperingand dilatation(arrow).

B: Left hydronephrosis

\section{Stage: Cervical Squamous Cell Carcinoma.}

CT stage: IVA

EUA Stage: IV A 


\section{Sameh Mohamed Abdel Wahab, et al.,}

Case no.3:35 year old female

Premenopausal, a known case of cervical squamous cell cancinoma post combined chemo and radiotherapy with recurrent bleeding.

\section{Post contrast CT examination:}

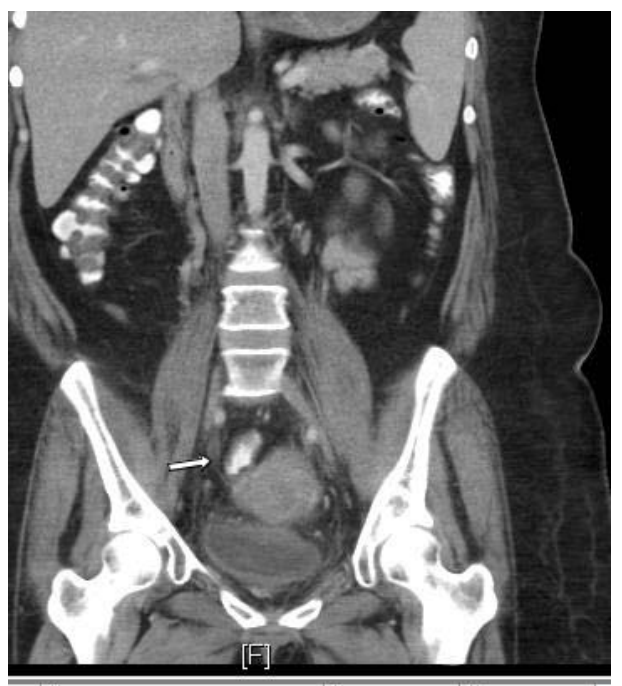

Figure (4): Coronal image demonstrates ureteric entrapment within the mass(white arrow). The ureter is not opacified

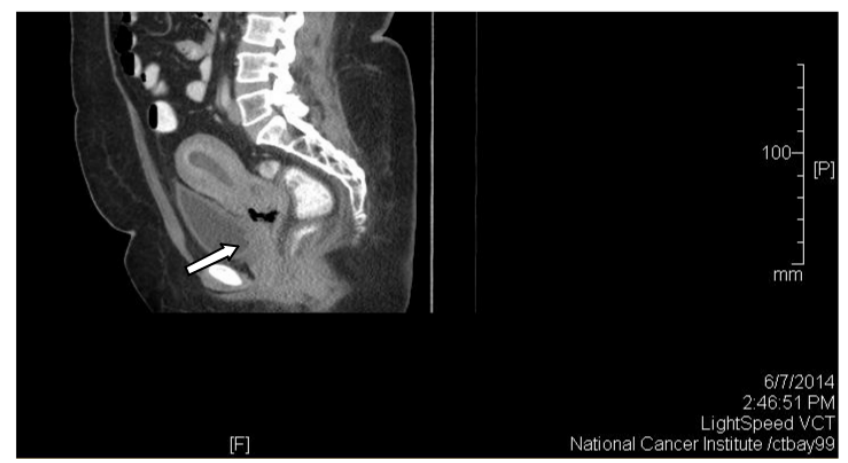

Figure (5): Sagittal image demonstrating irregular mass invading bladder wall posteriorly(white arrow), and invading upper vagina.EUA and cystoscopy revealed free bladder, while pathology proved invasion of the muscle layer of the bladder.

\section{Staging: Recurrent cervical Squamous cell carcinoma:}

CT stage: IVA

Pathology Stage: IVA

EUA Stage: IIIA

Note: No pelvic lymph nodes were detected in CT, while pathology revealed invasion of one subcentimetric iliac lymph node. 


\section{DISCUSSION:}

Endometrial cancer staging is mainly surgical, however with the aid of radiological imaging together with the tumour grade determined by histopathological analysis of endometrial biopsy; the risk of the tumour whether high, intermediate or low could be determined preoperatively. This is important to determine whether patients are treated at a local centre (those with low and intermediate risk) or a specialist gynaecologic oncology centre (those with high risk) $^{(11)}$.

The aim of this work was not to prove that CT is the best modality for local staging of uterine malignancies. The aim was to get the maximum benefit of a procedure which is supposed to be performed routinely for diagnosed cancer patients in order to detect distant metastasis. Thus if we could prove that CT could provide relatively reliable results for local staging this would add benefit to a procedure which is already performed.

\section{Endometrial Carcinoma:}

In a study by Tsili and Colleagues 21 women with newly diagnosed endometrial carcinoma examined by 16-row CT scanner. Reformatted CT images had accuracy of $95 \%$, in evaluating myometrial invasion and $81 \%$, in assessing cervical infiltration. Compared to $75 \%$ and $91 \%$ in our study respectively. The small sample size in our study is predicted to be the cause of the discrepancy in accuracy values compared to Tsili and Colleagues ${ }^{(12)}$.

Another comparable study for 25 women showed CT accuracy of 52\% for detection of deep myometrial invasion and $62.5 \%$ for cervical involvement. This study implemented axial CT images only in comparison to our study.

In this study differentiation between endometrium and inner myometrium couldn't be performed, yet fortunately the changes which were applied in the FIGO staging system in year 2009 aided in improving our CT results. Stage I was no longer divided to $\mathrm{A}, \mathrm{B}$ and $\mathrm{C}$, only $\mathrm{A}$ and $\mathrm{B}$. Thus according to the new classification, there is no value of differentiating endometrium from inner myometrium.

In our study a sample of 18 patients was subjected to a triphasic post contrast analysis. We found out that there was no added benefit in using this technique regarding differentiation between endometrium and myometrium.

The overall accuracy of cervical invasion in our study was $91 \%$, with only one case showing false negative result. In this case the mass was a high fundal mass far away from the cervix.

In a study performed by Milam and Colleagues, women were identified as being at low risk for nodal metastasis based on three specific criteria on the final pathology reports:

1) Less than $50 \%$ myometrial invasion,

2) Tumor size less than $2 \mathrm{~cm}$, and

3) Well or moderately differentiated endometrioid histology. If the uterine specimen did not meet all three criteria, it was viewed as high risk for nodal metastasis. The study included 971 women. High-risk patients had 6.3 times the risk of nodal metastasis ${ }^{(13)}$.

\section{Cervical Cancer:}

In current work we were guided by Tsili and Colleagues (Tsili et al.,2013) in the assessment of the parametrial invasion, categorization of pelvic lymph nodes; whether abnormal or not and characterization of stage IA carcinoma. Their study involved 18 patients and used the same measures of this study in assessing parametrial and pelvic nodes invasion. When the neoplasm was not detected at all, it was characterized as stage $\mathrm{IA}^{(14)}$. 
The aforementioned study (Tsili et al.,2013) results showed overall accuracy of staging cervical cancer of $83 \%$ compared to $61.5 \%$ (EUA and pathology) to $66.7 \%$ (compared to pathology only) in our study. Accuracy of parametrial invasion was $92 \%$ compared to $61.5 \%$ (EUA and pathology) to $77.8 \%$ (compared to pathology alone) in our study.

In our study a trial was made, where CT examination was extended to include a triphasic post contrast examination, there was no added value in the accuracy of staging apart from delayed scans which provided much more concise images of the periureteral fat and better anatomical delineation, thus decreasing interobserver variability and aided in determining parametrial invasion with an estimated accuracy that was raised from $61.5 \%$ to $72.7 \%$.

In our study proper assessment of vaginal invasion was inaccurate due to difficulty with the anatomical demarcation which caused a high interobserver variability causing a decrease of the overall accuracy of staging. This agrees with Koyama and Colleagues which stated that regarding vaginal involvement, $\mathrm{CT}$ and MRI did not show benefit over pelvic examination for assessing vaginal invasion ${ }^{(15)}$.

As for lymph nodes metastases, in our study there were false positive and negative results regarding lymph nodes invasion, this was due to relying merely on the size for evaluation. When compared to pathological results it was proven that the size is not a very accurate measure, some large nodes proved to be hyperplastic while a subcentimetric node was metastatic.

An issue has been raised in our study, which is lymph nodal assessment. The FIGO staging system doesn't include lymph nodes evaluation. This represent a major drawback in the FIGO staging system as some cases would have metastatic lymph nodes however they are dealt with as early cases ${ }^{(16)}$.

\section{Recurrent Cases:}

In our study 10 cases were of recurrent uterine malignancy. Accuracy of staging was $90 \%$, almost all cases were staged correctly without over or understaging. Two cases disagreed with EUA, the first one was due to presence of iliac node not detected by EUA. Another case carried the possibility of infiltration of muscular layer of the bladder (detected in CT as bladder infiltration), which was not detected by EUA and was not detected by cystoscopy.

Park and Colleagues investigated Imaging findingsof $\mathrm{CT}$ in 36 patients with recurrent uterine cervical cancer. Their Results showed an accuracy of $80.5 \%$.

Our results were better due to the use of multidetector CT, Park and Colleagues used a conventional CT machine with a slicethickness of $10 \mathrm{~mm},{ }^{(17)}$.

In our study six cases had tumour at the anatomical site of the uterus with a size ranging from 4.5 to $8 \mathrm{~cm}$. Two cases were early cases had unrecognized tumour by CT and the other two cases had no tumour at the primary site, but instead one case presented by metastatic lymph nodes and the other one by peritoneal deposits.

\section{Recommendations:}

A well established protocol for CT staging of uterine malignancies that include local opacification of the vagina and rectum to establish proper results.

- $\quad$ EUA should not be considered as a gold standard to be compared with. Surgical pathology should remain the only gold standard even if number of cases is decreased.

- Lymph nodal assessment should be included in Cervical cancer staging. 


\section{REFERENCES:}

1. Jemal A., Siegel R., Ward E., Murray T., Xu J., Thun MJ. (2007) Cancer statistics, (2007): CA Cancer J., 57:43-66.

2. Jemal A., Siegel R., Ward E., Hao Y, Xu J, Murray T. and Thun MJ (2008): Cancer statistics, 2008. CA Cancer J. ,58 (2):71-96.

3. Balleyguier C., Sala E., Bergman A., Forstner R., Lopez C., Togashi K. et al.(2010): Staging of uterine cervical cancer with MRI: guidelines of the European Society of Urogenital Radiology. Eur Radiol. 21(5):1102-1110

2. Abd El All H.S. Refaat A. and Dandash K. (2007) Prevalence of Cervical neoplastic lesions and Human Papilloma Virus infection in Egypt: National Cervical Cancer Screening Project. Infect Agent Cancer.2: 12.

3. Odicini F, Tisi $G$, Rampinelli F, Miscioscia $R$, Sartori E \& Pecorelli S (2007): New development of the FIGO staging system. Gynecol Oncol ,107(1 Suppl 1):S8-S9.

4. Picorelli S (2009): Revised FIGO staging for carcinoma of the vulva, cervix, and endometrium. Int $J$ Gynaecol Obstet 105 (2): 103-4.

5. Tsili AC, Tsangou V, Koliopoulos G, Stefos T, and Argyropoulou M (2013): Early-stage cervical carcinoma: The role of multidetector CT in correlation with histopathological findings/ Journal of obstetrics and gynecology; 33(8):882-887

8. Darcy J.W (2009): Local staging of endometrial carcinoma: role of multidetector CT, invited commentary. RadioGraphics,. 29:2003-2005.

9. Cohen P, Tan AL, Penman A.(2009): The multidisciplinary tumour conference in gynecologic oncology: does it alter management? Int J Gynecol Cancer ,19 (9):1470-1472.

10. Tsili AC, Tsampoulas C, Dalkalitsis $N$, Stefanou D, Paraskevaidis E and Efremidis SC. (2008) Local staging of endometrial carcinoma: role of multidetector CT Eur Radiol. May,18(5):1043-8.

11. Hardesty LA., Sumkin JH, Hakim C et al. (2001): The ability of helical CT to preoperatively stage Endometrial Carcinoma. AJR Am Roentgenol 137:551554.

12. Trein K.,Holund B.and Mogansen $O$. (2007): Accuracy of preoperative tumor grade and intraoperative gross examination of myometrial invasion in patients with endometrial cancer. Acta Obstet Gynecol Scand, 86:739-741.

13. Milam M.R., Java J., Walker J.L., Metzinger D.S., Parker L.P. and Coleman R.L.(2012): Nodal Metastasis Risk in Endometrioid Endometrial Cancer. Obstet Gynecol, Feb ;119(2 Pt 1): 286- 292.

14. Tsili AC, Tsangou V, Koliopoulos G, Stefos T, and Argyropoulou M (2013): Early-stage cervical carcinoma: The role of multidetector CT incorrelation with histopathological findings/ Journal of obstetrics and gynecology; 33(8):882-887

15. Koyama T, Tamai K, Togashi K (2007): Staging of carcinoma of the uterine cervix and endometrium. Eur Radiol, 17: 2009-19.

16. Petsokseri J., Jaishuen A., Pattaranutaporn P., Jaishuen A. and Chansilpa Y (2012): Advanced Imaging Applications for Locally Advanced Cervical Cancer. Asian Pacific J Cancer Prev;13: 1713-1718.

17. Park D.H., Kim K.H.,Park S.Y., Lee B.H., Choi C.W. and Chin S.Y.(2000): Diagnosis of Recurrent Uterine Cervical Cancer: Computed Tomography versus Positron Emission Tomography. Korean J Radiol. Jan-Mar; 1(1): 51-55. 


\section{دور الأشعه المقطعية متعدده الثرائح فى التشخيص المرحلى لأورام الرحم الخبيثة}

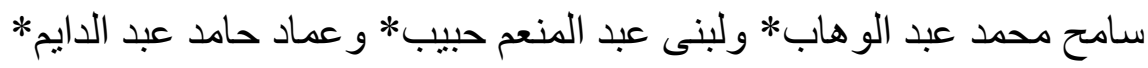

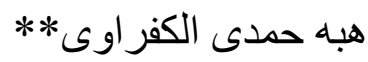

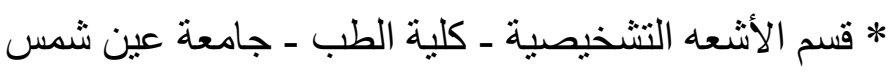

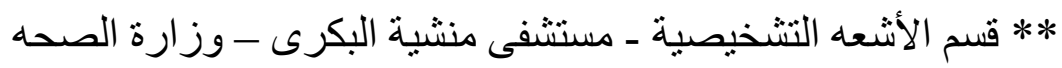

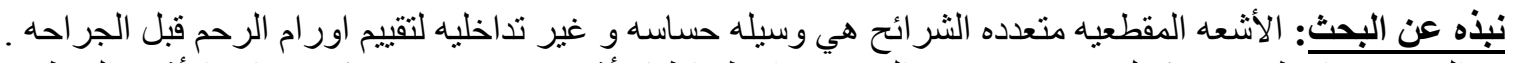

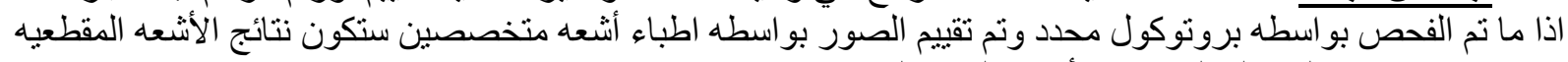

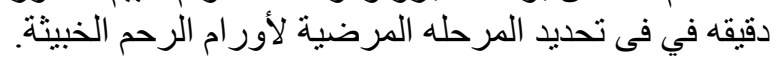

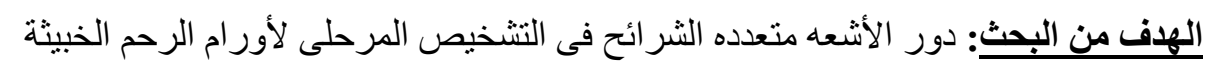

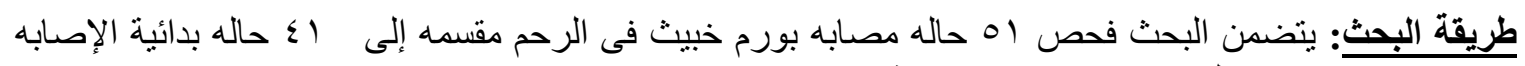
بالورم متضمنه جسم و وعنق الرحم و و الب حالات منتكسة الإصابه.

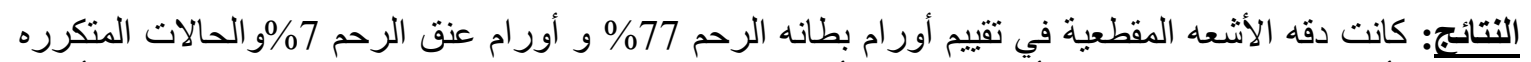

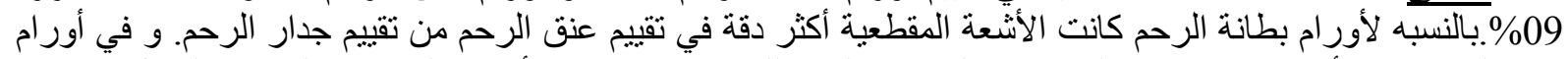

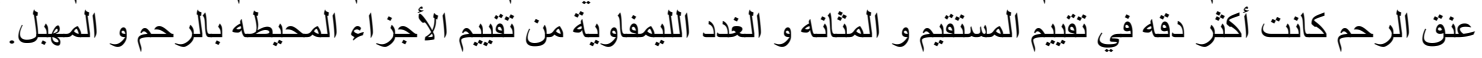

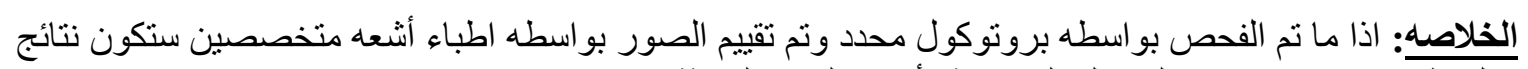
الأشعه المقطعيه دقيقه فى تحديد المرحله المرضية لأورام الرحم الخيبيثة. 